\title{
openheart Prognostic importance of the Controlling Nutritional Status (CONUT) score in patients undergoing cardiac resynchronisation therapy
}

\author{
Yukitoshi Ikeya, Yuki Saito (D) , Toshiko Nakai, Rikitake Kogawa, Naoto Otsuka, \\ Yuji Wakamatsu, Sayaka Kurokawa, Kimie Ohkubo, Koichi Nagashima, \\ Yasuo Okumura
}

\begin{abstract}
- Additional supplemental material is published online only. To view, please visit the journal online (http://dx.doi.org/10. 1136/openhrt-2021-001740).
\end{abstract}

To cite: Ikeya Y, Saito Y, Nakai T, et al. Prognostic importance of the Controlling Nutritional Status (CONUT) score in patients undergoing cardiac resynchronisation therapy. Open Heart 2021;8:e001740. doi:10.1136/ openhrt-2021-001740

Received 30 May 2021 Accepted 14 0ctober 2021

\section{Check for updates}

(C) Author(s) (or their employer(s)) 2021. Re-use permitted under CC BY-NC. No commercial re-use. See rights and permissions. Published by BMJ.

Department of Medicine, Nihon University School of Medicine Graduate School of Medicine, Itabashi-ku, Tokyo, Japan

Correspondence to Dr Yuki Saito; saito.yuki@nihonu.ac.jp

\section{ABSTRACT}

Aims Malnutrition is common and associated with worse clinical outcomes in patients with heart failure (HF). The Controlling Nutritional Status (CONUT) score is an integrated index for evaluating diverse aspects of the complex mechanism of malnutrition. However, the relationship between the severity of malnutrition assessed by the CONUT score and clinical outcomes of HF patients receiving cardiac resynchronisation therapy (CRT) has not been fully clarified.

Methods Clinical records of 263 patients who underwent pacemaker or defibrillator implantation for CRT between March 2003 and October 2020 were retrospectively evaluated. The CONUT score was calculated from laboratory data obtained before CRT device implantation. Patients were divided into three groups: normal nutrition (CONUT scores $0-1, n=58$ ), mild malnutrition (CONUT scores $2-4, n=132$ ) and moderate or severe malnutrition (CONUT scores $5-12, n=73$ ). The primary endpoint was all-cause mortality.

Results The moderate or severe malnutrition group had a lower body mass index, more advanced New York Heart Association functional class, higher Clinical Frailty Scale score, lower levels of haemoglobin and higher levels of $\mathrm{N}$-terminal probrain natriuretic peptide (all $\mathrm{p}<0.05$ ). In the moderate or severe malnutrition group, the CRT response rate was significantly lower than for the other two groups $(\mathrm{p}=0.001)$. During a median follow-up period of 31

(10-67) months, 103 (39.1\%) patients died. Kaplan-Meier analysis revealed that the moderate or severe malnutrition group had a significantly higher mortality rate (log-rank $p<0.001)$. A higher CONUT score and CONUT score $\geq 5$ remained significantly associated with all-cause mortality after adjusting for previously reported clinically relevant factors and the conventional risk score (VALID-CRT risk score) (all $p<0.05$ ).

Conclusions A higher CONUT score before CRT device implantation was strongly associated with HF severity, frailty, lower CRT response rate and subsequent long-term all-cause mortality.

\section{INTRODUCTION}

Heart failure (HF) is a major cause of death and hospital admission and has become a

\section{Key questions}

What is already known about this subject?

- Malnutrition is a common condition associated with worse clinical outcomes in patients with heart failure (HF). The Controlling Nutritional Status (CONUT) score is an integrated index for evaluating diverse aspects of the complex mechanism of malnutrition. However, the relationship between the severity of malnutrition assessed by the CONUT score and clinical outcomes of HF patients with cardiac resynchronisation therapy (CRT) has not been fully clarified.

What does this study add?

- A higher CONUT score before CRT device implantation was strongly associated with HF severity, frailty, lower CRT response rate and subsequent long-term all-cause mortality.

How might this impact on clinical practice?

- Our findings indicate that a simple and objective nutritional assessment using the CONUT score should be considered at the time of CRT device implantation. Patients with moderate or severe malnutrition might require more intensive follow-up or more aggressive management to improve their clinical outcomes.

significant public health problem. ${ }^{1}$ Cardiac resynchronisation therapy (CRT) is an effective treatment in addition to optimal medication therapy for patients with advanced-staged $\mathrm{HF}$ with reduced ejection fraction $(\mathrm{EF}) .^{23}$ CRT has been shown to reduce mortality and improve functional capacity in most eligible HF patients. ${ }^{4}$ However, $20 \%-30 \%$ of patients fail to respond to CRT, and long-term death rates remain high. ${ }^{5}$ Therefore, there is an unmet clinical need for early identification of CRT non-response and long-term poor clinical outcomes.

Several recent studies report that malnutrition is one of the most important determinants 
of poor clinical outcomes in $\mathrm{HF}^{6}$ Low nutritional intake due to intestinal oedema, anorexia, liver dysfunction and chronic inflammation all result in HF-related malnutrition. $^{78}$ The Controlling Nutritional Status (CONUT) score is one of several nutritional indices and is reported to be one of the most robust markers of nutritional status. ${ }^{9}$ The CONUT score is calculated from the serum albumin level, total cholesterol and total peripheral lymphocyte count, encompassing an immune-nutritional index that reflects protein/lipid metabolism and immunocompetence. Malnutrition assessed by the CONUT score has been shown to be related to worse clinical outcomes in HF patients. ${ }^{10} 11$ However, the relationship between the severity of malnutrition assessed by the CONUT score and clinical outcomes of HF patients receiving CRT has not been fully clarified. Thus, this real-world observational study aimed to clarify the relationship between nutritional status assessed by the CONUT score and CRT response and long-term mortality in CRT recipients.

In recent years, the number of patients needing CRT has been increasing alongside the growing older adult population, as the prevalence of HF rises sharply with age. ${ }^{1}$ Because older adults have many comorbidities and heterogeneous pathology, the role of nutritional status may be a pivotal factor in predicting clinical outcomes. ${ }^{12} 13$ Therefore, we also investigated whether the CONUT score would yield predictive values for both adult ( $<75$ years) and older adult ( $\geq 75$ years) CRT recipients.

\section{METHODS}

\section{Patients and study protocol}

This is a single-centre, retrospective, observational study of consecutive patients who underwent CRT device implantation at Nihon University Itabashi Hospital between March 2004 and October 2020. A total of 285 patients were reviewed, and 23 patients were excluded due to lack of data for one or more of the components to the CONUT score (serum albumin level, total cholesterol level and total peripheral lymphocyte count). A final 263 patients were included in this study.

\section{Nutritional assessment using the CONUT score and assessment of frailty}

The CONUT score was calculated from three variables (serum albumin level, total cholesterol level and total peripheral lymphocyte count) obtained before CRT device implantation. ${ }^{14}$ The range of the CONUT score is $0-12$, with higher values indicating worse nutritional status. Patients with CONUT scores $0-1$ have a normal nutritional status, those with CONUT scores of 2-4 have a mild degree of malnutrition, those with CONUT scores of 5-8 have a moderate degree of malnutrition, and CONUT scores of 9-12 indicate a severe degree of malnutrition. ${ }^{14}$ We divided patients into three groups, accordingly: normal nutritional group (CONUT scores $0-1, \mathrm{n}=58$ ), mild malnutrition (CONUT scores 2-4, $\mathrm{n}=132$ ) and moderate or severe malnutrition (CONUT scores 5-12, n=73). The Clinical Frailty Scale was used to measure the level of frailty for patients before implantation of a CRT device. ${ }^{15}$ This scale is based on clinical judgement using available clinical information.

\section{Echocardiography}

Echocardiographic measurements were routinely performed before CRT device implantation by experienced echocardiographers. These measurements were made according to the American Society of Echocardiography guidelines. ${ }^{16}$ Left ventricular (LV) end-systolic and end-diastolic volumes were measured in the apical fourchamber and two-chamber views. LVEF was measured by the modified Simpson method. Mitral regurgitation (MR) was graded on a 4-point scale using a colour flow Doppler image.

\section{Device therapy}

CRT-pacing (CRT-P) or CRT-defibrillation (CRT-D) device implantation was performed under local anaesthesia using standard transvenous techniques. The LV lead was inserted into the coronary sinus and advanced into the posterolateral $\mathrm{LV}$ site. The right ventricle (RV) and right atrial leads were inserted into the RV apex and right atrial appendage, respectively. Next, patients were follow-up in dedicated device therapy clinics at regular 3-month or 6-month intervals.

\section{Follow-up and endpoint}

The study endpoint was all-cause mortality. All patients were followed from the date of device implantation till December 2020 or death. Follow-up data were obtained in a blinded manner via a review of medical records. We evaluated two definitions of CRT response: functional and echocardiographic. Functional CRT response was defined as the combination of improved functional status in at least one New York Heart Association (NYHA) functional class and the absence of death or hospitalisation of $\mathrm{HF}$ at 6 months after CRT device implantation. ${ }^{17}{ }^{18}$ Echocardiographic CRT response was defined as an improvement in LVEF of at least $5 \%$ or a reduction in LV endsystolic volume (LVESV) of at least $15 \%$ at 6 months after CRT implantation.

\section{Statistical analysis}

Categorical variables are presented as numbers (percentage) and continuous variables as the median (IQR). Statistical differences among categorical variables were compared by the $\chi^{2}$ test with Bonferroni correction. Continuous variables were compared using one-way analysis of variance followed by the post hoc Turkey-Kramer test, or the Kruskal-Wallis test followed by the Steel-Dwass test for non-symmetrical continuous variables.

Survival was assessed using the Kaplan-Meier method, with differences compared by the log-rank test. Next, univariate and multivariate Cox proportional hazards regression analyses were performed to analyse the association between the CONUT score (continuous variables, and moderate or severe malnutrition vs normal or 
mild malnutrition) and all-cause mortality. N-terminal probrain natriuretic peptide (NT-pro-BNP) data were subjected to natural $\log$ transformation (ln) to meet model assumptions. HRs with $95 \%$ CIs were calculated.

Using the multivariate Cox proportional hazards regression analysis, we constructed multivariate models to adjust for the effects of established clinically relevant factors including age, sex, ischaemic aetiology, atrial fibrillation, QRS duration $>150 \mathrm{~ms}$, LVESV, LVEF, moderate or severe MR, renal function, statin use and acute decompensated HF (ADHF) at admission (model 1), to adjust for the effects of the conventional VALID-CRT risk score (model 2 ), and to adjust for liver function parameters including total bilirubin, aspartate aminotransferase, alanine aminotransferase and $\gamma$-glutamyl transferase (model 3). The VALID-CRT risk score was constructed and validated using the following variables: age, sex, implantable cardioverter defibrillator back-up, atrial fibrillation, presence or absence of atrioventricular junction ablation in the case of atrial fibrillation, ischaemic aetiology, diabetes mellitus, NYHA class and LVEF. ${ }^{19}$

To further evaluate the association of the CONUT score and mid-term mortality risk, the predictive capacity of the CONUT score to identify patients at the risk of 1-year allcause mortality following CRT device implantation was assessed by receiver operating characteristic (ROC) curve analyses. In addition, to evaluate whether the accuracy of predicting 1-year all-cause mortality would improve after adding the CONUT score to a baseline model of the VALID-CRT risk score, the C-statistics, net reclassification improvement and integrated discrimination improvement were calculated.

Statistical analyses were conducted using JMP V.13.0 (SAS Institute) and the R Statistics V.3.5.3 (R Foundation for Statistical Computing, Vienna, Austria). A $p<0.05$ was considered statistically significant.

\section{RESULTS}

\section{Baseline patient characteristics}

The distribution of the CONUT scores is shown in online supplemental figure 1 . The median (IQR) CONUT score was 3 (2-5). Among 263 patients, $73(27.7 \%)$ patients presented a CONUT score $\geq 5$, suggestive of moderate or severe degree of malnutrition. The baseline clinical characteristics of each group are shown in table 1 . The moderate or severe malnutrition group had lower body mass indices (BMIs), a more advanced NYHA functional class, higher prevalence of ADHF at the time of admission, and higher clinical frailty scale scores. The CONUT score significantly correlated with the clinical frailty scale score $(\mathrm{r}=0.27, \mathrm{p}<0.001)$. For laboratory data, the moderate or severe malnutrition group had lower levels of haemoglobin and estimated glomerular filtration rate (eGFR) and higher levels of blood urea nitrogen (BUN), aspartate aminotransferase and NT-pro-BNP. Among 263 patients, $98(37.2 \%)$ patients receiving a stain for dyslipidaemia. The prevalence of stain usage did not differ among three groups $(\mathrm{p}=0.69)$. The median (IQR) CONUT score did not differ significantly between the patients underwent CRT-P implantation $(\mathrm{n}=98)$ and those underwent CRT-D implantation $(\mathrm{n}=165)(3(2-4)$ vs $3(2-5), \mathrm{p}=0.82)$.

\section{Nutritional status and CRT response}

In this study population, response to functional CRT was evaluated in 257 patients at 6 months after CRT device implantation. Among these patients, 191 (74.3\%) patients were classified as functional CRT responders. The response rates were $83.6 \%$ in the normal nutritional group, $78.8 \%$ in the mild malnutrition group and $58.5 \%$ in the moderate or severe malnutrition group. The CRT response rate was significantly lower in the moderate or severe malnutrition group than the other two groups $(\mathrm{p}=0.001)$. In addition, 244 patients underwent 6-month echocardiographic follow-up, of whom 178 (72.9\%) were classified as echocardiographic CRT responders. The echocardiographic CRT response rate was $83.3 \%$ in the normal nutrition group, $73.1 \%$ in the mild malnutrition group and $64.1 \%$ in the moderate or severe malnutrition group $(\mathrm{p}=0.051)$. Non-echocardiographic responders had significantly higher CONUT score (the median (IQR)) than echocardiographic CRT responders (4 (3-6) vs $2(1-4), \mathrm{p}<0.001)$.

\section{Nutritional status and clinical outcome}

During a median follow-up period of 31 (10-67) months, $103(39.6 \%)$ of the total of 263 patients died. Cardiac deaths occurred in 57 patients (HF death, $\mathrm{n}=41$; ventricular fibrillation, $\mathrm{n}=9$; sudden cardiac death, $\mathrm{n}=6$; and acute myocardial infarction, $\mathrm{n}=1$ ). Non-cardiac deaths occurred in 46 patients (infection or sepsis, $\mathrm{n}=13$; cancer, $\mathrm{n}=8$; cerebral haemorrpage, $\mathrm{n}=5$; respiratory diseases, $n=3$, and other causes of death, $n=17$ ). One device infection occurred and lead extraction was performed in the follow-up period. The Kaplan-Meier analysis found that the moderate or severe malnutrition group had a significantly higher mortality rate (log-rank test: $\mathrm{p}<0.001$, figure 1$)$. In the subanalysis, 180 patients were younger adults ( $<75$ years old), and 83 patients were older adults ( $\geq 75$ years old). The Kaplan-Meier analysis of the subgroups showed that the moderate or severe malnutrition group had a higher occurrence of all-cause mortality in both the younger adult population (log-rank test: $\mathrm{p}<0.001)$ and the older adult population $(\mathrm{p}=0.024)$ (figure 2). All-cause mortality did not differ significantly between patients with ischaemic aetiology $(\mathrm{n}=177)$ vs non-ischaemic aetiology $(\mathrm{n}=86)(\mathrm{p}=0.56)$. The univariate Cox proportional hazards regression analysis showed that both a higher CONUT score (continuous variable) and a CONUT score $\geq 5$ (vs normal or mild malnutrition) were significantly associated with all-cause mortality, along with a lower BMI, higher NYHA functional class, atrial fibrillation, QRS duration, lower haemoglobin and eGFR, higher BUN and ln (NT-pro-BNP), and a lower LVEF (online supplemental table 1). A decrease in any parameter of the CONUT score (lymphocyte count, albumin 
Table 1 Clinical characteristics of patients stratified into three groups according to nutritional status

\begin{tabular}{|c|c|c|c|c|}
\hline Item & $\begin{array}{l}\text { Normal nutrition CONUT } \\
\text { score } \leq 1(n=58)\end{array}$ & $\begin{array}{l}\text { Mild malnutrition } 2 \leq \text { CONUT } \\
\text { score } \leq 4(n=132)\end{array}$ & $\begin{array}{l}\text { Moderate or severe malnutrition } \\
\text { CONUT score } \geq 5(n=73)\end{array}$ & $P$ value \\
\hline \multicolumn{5}{|l|}{ Baseline clinical data } \\
\hline Age, years & $67(57-75)$ & $69(60-77)$ & $72(63-78)$ & 0.22 \\
\hline Male & $37(63.8)$ & $107(81.1)^{\star}$ & $58(79.5)$ & 0.036 \\
\hline Body mass index, $\mathrm{kg} / \mathrm{m}^{2}$ & $22.3(20.2-25.3)$ & $22.7(20.3-25.5)$ & $20.9(18.8-23.1) \dagger$ & 0.008 \\
\hline \multicolumn{5}{|l|}{ NYHA class } \\
\hline$\|$ & $12(30.7)$ & $21(15.7)$ & $6(8.2)$ & 0.11 \\
\hline III & $40(70.1)$ & $102(76.6)$ & $44(60.2) \dagger$ & 0.046 \\
\hline IV & $5(8.6)$ & $10(7.6)$ & $23(31.5)^{\star} \dagger$ & $<0.001$ \\
\hline Clinical Frailty Scale & $4(4-5)$ & $4(4-5)$ & $4(4-5)^{*} \dagger$ & 0.004 \\
\hline Diabetes mellitus & $14(24.1)$ & $50(37.9)$ & $36(49.3)^{\star}$ & 0.013 \\
\hline Hypertension & $27(46.6)$ & $72(54.5)$ & $36(49.3)$ & 0.55 \\
\hline COPD & $4(6.9)$ & $3(2.3)$ & $1(1.4)$ & 0.19 \\
\hline Haemodialysis & $1(1.7)$ & $6(4.5)$ & $6(8.2)$ & 0.22 \\
\hline Ischaemic aetiology & $14(24.1)$ & $46(34.8)$ & $26(35.6)$ & 0.29 \\
\hline Atrial fibrillation & $9(15.5)$ & $43(32.6)^{\star}$ & $13(17.8)$ & 0.012 \\
\hline QRS duration, ms & $150(124-167)$ & $150(126-174)$ & $148(127-167)$ & 0.78 \\
\hline VALID-CRT risk score & $0.55(-0.10-1.02)$ & $0.91(0.20-1.44)$ & $0.89(0.18-1.52)$ & 0.048 \\
\hline $\begin{array}{l}\text { Acute decompensated heart failure at } \\
\text { admission }\end{array}$ & $19(32.7)$ & $59(44.7)$ & $49(67.1)^{\star} \dagger$ & $<0.001$ \\
\hline \multicolumn{5}{|l|}{ Medications } \\
\hline ACE-I or ARB & $46(79.3)$ & $78(59.1)^{\star}$ & $50(69.4)$ & 0.020 \\
\hline$\beta$-blocker & $57(98.2)$ & $117(88.6)$ & $66(91.7)$ & 0.088 \\
\hline Diuretics & $56(96.6)$ & $116(87.9)$ & $61(84.7)$ & 0.088 \\
\hline Statins & $19(32.7)$ & $50(37.8)$ & $29(39.7)$ & 0.69 \\
\hline \multicolumn{5}{|l|}{ Laboratory data } \\
\hline Haemoglobin, g/L & $136(123-149)$ & $128(114-137)^{\star}$ & $110(93-126)^{\star} \dagger$ & $<0.001$ \\
\hline White cell count, $\times 10^{9} / \mathrm{L}$ & $6.8(5.5-8.2)$ & $6.0(4.9-7.4)^{*}$ & $6.6(5.0-8.0)$ & 0.041 \\
\hline Lymphocyte count, $\times 10^{9} / \mathrm{L}$ & $1.8(1.5-2.1)$ & $1.1(1.0-1.5)^{\star}$ & $0.9(0.6-1.1)^{\star} \dagger$ & $<0.001$ \\
\hline BUN, mg/dL & $20.3(15.4-26.5)$ & $22.9(17.7-29.2)$ & $24.8(19.2-38.1)^{\star}$ & 0.009 \\
\hline $\mathrm{Cr}, \mathrm{mg} / \mathrm{dL}$ & $1.0(0.8-1.1)$ & $1.0(0.9-1.6)^{\star}$ & $1.2(0.9-1.8)^{\star}$ & 0.002 \\
\hline eGFR, ml/min/1.73 m² & $56.5(39.8-70.1)$ & $53.2(34.2-65.3)$ & $44.5(28.5-57.8)^{\star}$ & 0.007 \\
\hline Total bilirubin, $\mathrm{mg} / \mathrm{dL}$ & $0.6(0.4-0.8)$ & $0.6(0.4-0.9)$ & $0.6(0.4-0.9)$ & 0.47 \\
\hline AST, U/L & $23(18-29)$ & $22(17-30)$ & $27(21-36)^{*} \dagger$ & 0.037 \\
\hline ALT, U/L & $19(13-33)$ & $18(12-28)$ & $20(13-36)$ & 0.46 \\
\hline GGT, U/L & $37(21-69)$ & $46(24-88)$ & $53(28-99)$ & 0.25 \\
\hline Albumin, $\mathrm{g} / \mathrm{dL}$ & $4.0(3.7-4.2)$ & $3.7(3.5-4.0)^{\star}$ & $2.9(2.6-3.2)^{*} \dagger$ & $<0.001$ \\
\hline Total cholesterol, mg/dL & $190(170-216)$ & $159(134-191)^{\star}$ & $138(118-163)^{\star} \dagger$ & $<0.001$ \\
\hline NT-pro-BNP, pg/mL & 2031 (960-3703) & $2733(1484-6597)^{\star}$ & $6111(1983-12949)^{\star} \dagger$ & $<0.001$ \\
\hline \multicolumn{5}{|l|}{ Echocardiographic data } \\
\hline LVEDV, mL & $218(149-262)$ & $202(160-262)$ & $197(150-244)$ & 0.61 \\
\hline LVESV, mL & $125(67-168)$ & $148(100-197)$ & $147(97-185)$ & 0.65 \\
\hline LVEF, \% & $29(23-36)$ & $30(22-37)$ & $28(22-38)$ & 0.78 \\
\hline Moderate or severe MR & $5(8.9)$ & $15(11.9)$ & $12(16.9)$ & 0.39 \\
\hline
\end{tabular}

Values are the median (IQR) or number (\%). For multiple comparisons, the ANOVA test was used for symmetrical continuous variables, the Kruskal-Wallis test for non-symmetrical continuous variables and the $\chi^{2}$ test for categorical variables. All pair comparisons were performed based on the Tukey-Kramer test for symmetrical continuous variables, the SteelDwass test for non-symmetrical continuous variables, and the $\chi^{2}$ test with Bonferroni correction for categorical variables.

${ }^{*} \mathrm{P}<0.05$ vs normal nutrition.

$\dagger P<0.05$ vs mild malnutrition.

ACE-I, ACE inhibitor; ALT, alanine aminotransferase; ANOVA, analysis of variance; ARB, angiotensin receptor blocker; AST, aspartate aminotransferase; BUN, blood urea nitrogen; CONUT, Controlling Nutritional Status; COPD, chronic obstructive pulmonary disease; Cr, creatinine; CRT, cardiac resynchronization therapy; eGFR, estimated glomerular filtration rate; GGT, $\gamma$-glutamyl transferase; LVEDV, left ventricular end-diastolic volume; LVEF, left ventricular ejection fraction; LVESV, left ventricular end-systolic volume; MR, mitral regurgitation; NT-pro-BNP, N-terminal probrain natriuretic peptide; NYHA, New York Heart Association. 


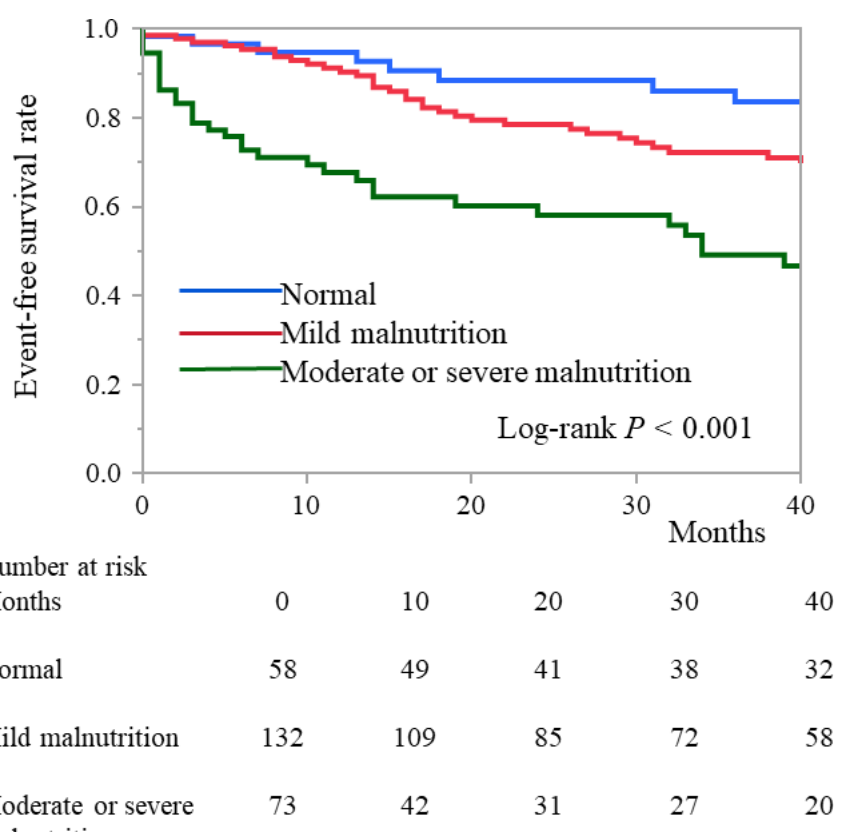

malnutrition

Figure 1 Kaplan-Meier curves of overall survival for patient groups defined according to nutritional status assessed by the CONUT score. CONUT, Controlling Nutritional Status.

and total cholesterol) was significantly associated with all-cause mortality (online supplemental table 1). In the multivariate Cox proportional hazards regression analysis, a higher CONUT score and a CONUT score $\geq 5$ (vs normal or mild malnutrition) remained significantly associated with all-cause mortality after adjusting for previously reported clinically relevant factors (age, sex, ischaemic aetiology, atrial fibrillation, QRS duration $>150$ ms, LVESV, LVEF, moderate or severe MR, eGFR, statin use and ADHF at admission), conventional risk score (VALID-CRT risk score) and liver function parameters (table 2).

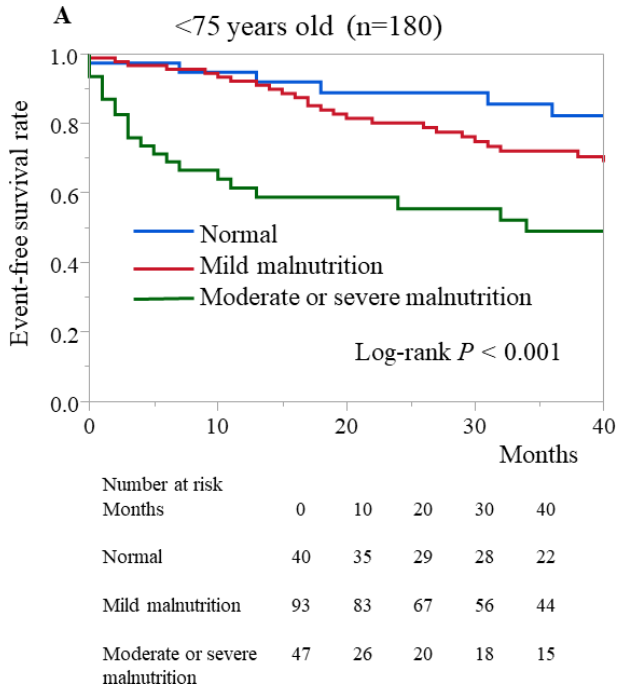

Discriminative power of CONUT score for assessing risk

We performed an ROC curve analysis to evaluate the predictive value of the CONUT score for 1-year all-cause mortality. The cut-off CONUT score value of 6 had a sensitivity of 0.59 and a specificity of 0.84 for predicting 1-year all-cause mortality (C-statistic=0.74). Furthermore, adding the CONUT score as a continuous variable to a baseline model with VALID-CRT risk score significantly increased the C-statistic, net reclassification improvement, and integrated discrimination improvement for predicting 1-year all-cause mortality (table 3).

\section{DISCUSSION}

The results of this study demonstrated a strong association between the severity of malnutrition (CONUT score) and a poor clinical outcome in CRT recipients. Our study had four major findings. First, a higher CONUT score was associated with a lower BMI, more severe HF, renal dysfunction, frailty and a lower CRT response rate. Second, a higher CONUT score before CRT device implantation was strongly associated with all-cause mortality following CRT device implantation, and this association persisted regardless of age group. Third, the CONUT showed an incremental prognostic value when added to the conventional risk score for predicting 1-year mortality.

In our study population, $27.7 \%$ of patients presented moderate or severe malnutrition. Malnutrition is common in HF patients and is associated with poor clinical outcomes. ${ }^{20}$ Intestinal oedema, affected by gastrointestinal congestion and low nutritional intake due to appetite loss, causes malabsorption, chronic inflammation, and a cytokine-induced hypermetabolic condition, ${ }^{21}$ which leads to a vicious cycle of inflammation, catabolic drive, and malnutrition. The CONUT score was developed for early detection of poor nutritional status. This score is an integrated index that reflects immunity status,

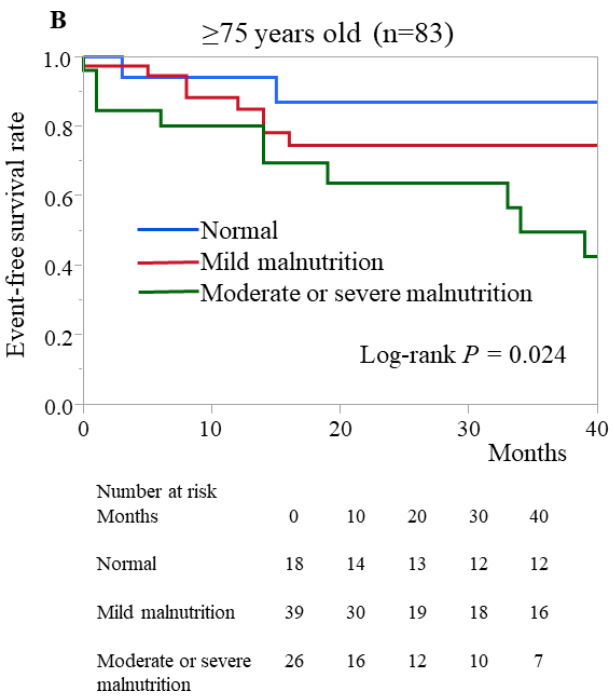

Figure 2 Kaplan-Meier curves of overall survival for patient groups defined according to nutritional status assessed by the CONUT score, divided according to age: $<75$ years old $(A)$ and $\geq 75$ years old $(B)$. CONUT, Controlling Nutritional Status. 


\begin{tabular}{|c|c|c|c|c|c|c|}
\hline \multirow[b]{2}{*}{ Model } & \multicolumn{3}{|c|}{ CONUT score (per one increase) } & \multicolumn{3}{|c|}{ CONUT score $\geq 5$} \\
\hline & HR & $95 \% \mathrm{Cl}$ & $P$ value & HR & $95 \% \mathrm{Cl}$ & $P$ value \\
\hline Model 1 & 1.25 & 1.14 to 1.37 & $<0.001$ & 2.23 & 1.37 to 3.60 & 0.001 \\
\hline Model 2 & 1.22 & 1.13 to 1.31 & $<0.001$ & 2.05 & 1.14 to 1.81 & $<0.001$ \\
\hline Model 3 & 1.24 & 1.13 to 1.35 & $<0.001$ & 2.03 & 1.26 to 3.22 & 0.003 \\
\hline
\end{tabular}

Model 1=adjusted for age, sex and clinically relevant factors (ischaemic aetiology, atrial fibrillation, QRS duration >150 ms, left ventricular end-systolic volume, left ventricular ejection fraction, moderate or severe MR, estimated glomerular filtration rate, statin use and acute decompensated heart failure at admission). Model 2=adjusted for VALID-cardiac resynchronisation therapy risk score. Model 3=adjusted for age, sex and liver function parameters (total bilirubin, aspartate aminotransferase, alanine aminotransferase, and $\gamma$-glutamyl transferase). CONUT, Controlling Nutritional Status.

protein reserve and lipid metabolism. Thus, our findings expand the data for this index regarding diverse aspects of the complex malnutrition mechanism in HF and build on the previously reported usefulness of the CONUT score for HF risk-stratification. ${ }^{9} 10$

Many studies have explored the association between clinical characteristics, echocardiographic parameters, CRT non-response and long-term mortality. ${ }^{22}{ }^{23}$ However, the relationship between the nutritional status of patients and the clinical outcomes of CRT recipients was not fully understood. Our results revealed that a higher CONUT score was associated with not only a lower CRT response rate but also 1-year and long-term all-cause mortality. These associations remained significant even after adjusting clinically relevant factors and liver function test parameters.

We found that a cut-off CONUT score value of 6 had a sensitivity of 0.59 and a specificity of 0.84 for predicting 1-year all-cause mortality. Moreover, the CONUT score showed an incremental prognostic value for predicting 1-year mortality over the valid cardiac resynchronisation therapy (VALID-CRT) risk score. Adding the CONUT score to a baseline model with VALID-CRT risk score significantly increased the C-statistic, net reclassification improvement, and integrated discrimination improvement for predicting 1-year all-cause mortality. Currently, the VALID-CRT risk score is a widely used and wellestablished comprehensive risk-stratification tool for CRT device implantation. ${ }^{19}$ However, the VALID-CRT risk score does not include the effect of nutritional status. Therefore, adding the CONUT score to the VALID-CRT risk score provides additional information, improving the prediction of poor clinical outcomes.

\section{Clinical implications}

Our findings indicate that a simple and objective nutritional assessment using the CONUT score should be considered before CRT device implantation. Patients with moderate or severe malnutrition might require more intensive follow-up or more aggressive management to improve their clinical outcomes, regardless of their age group. Therefore, early detection of malnutrition by the CONUT score and early nutritional care before and alongside CRT would benefit those receiving the therapy. In particular, nutritional interventions aiming to increase protein and energy intake for HF patients with malnutrition can improve nutritional status, hospital readmission rate and mortality. ${ }^{24}{ }^{25}$ One multicentre, randomised, controlled clinical trial reported that conventional HF treatment combined with an individualised nutritional intervention in malnourished hospitalised patients with HF reduced the risk of all-cause death or readmission for worsening of HF, with a maximum follow-up of 12 months. ${ }^{25}$

\section{Limitations}

This study has several limitations. First, it was a retrospective single-centre study with a relatively small sample size. Second, we could not compare with other clinical nutritional markers such as the Subjective Global Assessment and the Mini Nutritional Assessment because we could not obtain data on weight change, dietary intake change, gastrointestinal symptoms or physical information on muscle. ${ }^{26}{ }^{27}$ Finally, we only calculated the CONUT score before CRT device implantation. Therefore, the change in the CONUT score over time was not investigated. Further large-scale multicentre studies are needed to confirm our findings.

Table 3 Evaluation of the increased predictive ability of the CONUT score for 1-year all-cause mortality when added to the VALID-CRT risk score

\begin{tabular}{lllllll}
\hline Risk score & C-statistic $(\mathbf{9 5} \%$ CI) & P value & NRI (95\% CI) & P value & IDI (95\% CI) & P value \\
\hline VALID-CRT risk score & $0.63(0.54$ to 0.72$)$ & Ref. & & Ref. & Ref. \\
VALID-CRT risk score+ CONUT score & $0.78(0.70$ to 0.86$)$ & 0.003 & $0.78(0.45$ to 1.12$)$ & $<0.001$ & $0.12(0.06$ to 0.18$)$ & $<0.001$ \\
\hline
\end{tabular}

CONUT, Controlling Nutritional Status; CRT, cardiac resynchronisation therapy; IDI, integrated discrimination improvement; NRI, net reclassification improvement. 


\section{CONCLUSIONS}

A higher CONUT score before CRT device implantation was strongly associated with HF severity, frailty, lower CRT response rate and subsequent 1-year and long-term all-cause mortality. Our findings suggest that nutritional assessment using the CONUT score may improve the risk stratification of CRT recipients.

\section{Twitter Yuki Saito @YukiSai43824890}

Acknowledgements We would like to thank Mrs. Alexandar Wendy and Mr. John Martin for English language editing.

Contributors YI, YS and TN designed the work and drafted the manuscript, RK, NO, YW and SK corrected data, KO, KN and YO conducted data analysis. All authors contributed critical revisions to the manuscript and approved the final version for submission.YS is responsible for the overall content as guarantor.

Funding The authors have not declared a specific grant for this research from any funding agency in the public, commercial or not-for-profit sectors.

Competing interests TN received lecture fees from Abbott Medical and Medtronic Japan, and YO belongs to the endowed departments of Boston Scientific Japan, Abbott Medical Japan, Japan Lifeline, Medtronic Japan and Nihon Kohden.

Patient consent for publication Not applicable.

Ethics approval This study was approved by the ethics committee of Nihon University Itabashi Hospital (RK-210209-8). The investigation conformed with the principles outlined in the Declaration of Helsinki.

Provenance and peer review Not commissioned; externally peer reviewed. Data availability statement Data are available on reasonable request.

Open access This is an open access article distributed in accordance with the Creative Commons Attribution Non Commercial (CC BY-NC 4.0) license, which permits others to distribute, remix, adapt, build upon this work non-commercially, and license their derivative works on different terms, provided the original work is properly cited, appropriate credit is given, any changes made indicated, and the use is non-commercial. See: http://creativecommons.org/licenses/by-nc/4.0/.

ORCID iD

Yuki Saito http://orcid.org/0000-0002-8193-9575

\section{REFERENCES}

1 Ponikowski P, Voors AA, Anker SD, et al. 2016 ESC Guidelines for the diagnosis and treatment of acute and chronic heart failure: The Task Force for the diagnosis and treatment of acute and chronic heart failure of the European Society of Cardiology (ESC)Developed with the special contribution of the Heart Failure Association (HFA) of the ESC. Eur Heart J 2016;37:2129-200.

2 Cazeau S, Leclercq C, Lavergne T, et al. Effects of multisite biventricular pacing in patients with heart failure and intraventricular conduction delay. N Engl J Med 2001;344:873-80.

3 Cleland JGF, Daubert J-C, Erdmann E, et al. The effect of cardiac resynchronization on morbidity and mortality in heart failure. $N$ Engl $J$ Med 2005;352:1539-49.

4 Bristow MR, Saxon LA, Boehmer J, et al. Cardiac-resynchronization therapy with or without an implantable defibrillator in advanced chronic heart failure. N Engl J Med 2004;350:2140-50.

5 Cleland J, Freemantle N, Ghio S, et al. Predicting the long-term effects of cardiac resynchronization therapy on mortality from baseline variables and the early response a report from the CARE-HF (cardiac resynchronization in heart failure) trial. J Am Coll Cardiol 2008;52:438-45.

6 Sze S, Pellicori P, Kazmi S, et al. Prevalence and prognostic significance of malnutrition using 3 scoring systems among outpatients with heart failure: a comparison with body mass index. JACC Heart Fail 2018;6:476-86.

7 Poehlman ET, Scheffers J, Gottlieb SS, et al. Increased resting metabolic rate in patients with congestive heart failure. Ann Intern Med 1994;121:860-2.

8 Lin $\mathrm{H}$, Zhang $\mathrm{H}$, Lin Z, et al. Review of nutritional screening and assessment tools and clinical outcomes in heart failure. Heart Fail Rev 2016;21:549-65.

9 Nochioka K, Sakata Y, Takahashi J, et al. Prognostic impact of nutritional status in asymptomatic patients with cardiac diseases: a report from the CHART-2 study. Circ J 2013;77:2318-26.

10 Yoshihisa A, Kanno Y, Watanabe S, et al. Impact of nutritional indices on mortality in patients with heart failure. Open Heart 2018;5:e000730.

11 Nishi I, Seo Y, Hamada-Harimura Y, et al. Utility of nutritional screening in predicting short-term prognosis of heart failure patients. Int Heart J 2018;59:354-60.

12 Cereda E, Klersy C, Pedrolli C, et al. The geriatric nutritional risk index predicts Hospital length of stay and in-hospital weight loss in elderly patients. Clin Nutr 2015;34:74-8.

13 Cereda E, Pedrolli C, Zagami A, et al. Nutritional risk, functional status and mortality in newly institutionalised elderly. Br J Nutr 2013;110:1903-9.

14 Ignacio de Ulíbarri J, González-Madroño A, de Villar NGP, et al. CONUT: a tool for controlling nutritional status. first validation in a hospital population. Nutr Hosp 2005;20:38-45.

15 Rockwood K, Song X, MacKnight C, et al. A global clinical measure of fitness and frailty in elderly people. CMAJ 2005;173:489-95.

16 Lang RM, Bierig M, Devereux RB, et al. Recommendations for chamber quantification: a report from the American Society of echocardiography's guidelines and standards Committee and the chamber quantification writing group, developed in conjunction with the European association of echocardiography, a branch of the European Society of cardiology. J Am Soc Echocardiogr 2005; 18:1440-63.

17 Nakai T, Ikeya $\mathrm{Y}$, Kogawa $\mathrm{R}$, et al. What are the expectations for cardiac resynchronization therapy? A validation of two response definitions. J Clin Med 2021;10:514.

18 Bleeker GB, Bax JJ, Fung JW-H, et al. Clinical versus echocardiographic parameters to assess response to cardiac resynchronization therapy. Am J Cardiol 2006;97:260-3.

19 Gasparini M, Klersy C, Leclercq C, et al. Validation of a simple risk stratification tool for patients implanted with cardiac resynchronization therapy: the VALID-CRT risk score. Eur $J$ Heart Fail 2015;17:717-24.

20 Aquilani R, Opasich C, Verri M, et al. Is nutritional intake adequate in chronic heart failure patients? J Am Coll Cardiol 2003;42:1218-23.

21 Valentova M, von Haehling S, Bauditz J, et al. Intestinal congestion and right ventricular dysfunction: a link with appetite loss, inflammation, and cachexia in chronic heart failure. Eur Heart $J$ 2016;37:1684-91.

22 Gold MR, Thébault C, Linde C, et al. Effect of QRS duration and morphology on cardiac resynchronization therapy outcomes in mild heart failure: results from the resynchronization reverses remodeling in systolic left ventricular dysfunction (REVERSE) study. Circulation 2012;126:822-9.

23 van Bommel RJ, Borleffs CJW, Ypenburg C, et al. Morbidity and mortality in heart failure patients treated with cardiac resynchronization therapy: influence of pre-implantation characteristics on long-term outcome. Eur Heart J 2010;31:2783-90.

24 Habaybeh D, de Moraes MB, Slee A, et al. Nutritional interventions for heart failure patients who are malnourished or at risk of malnutrition or cachexia: a systematic review and meta-analysis. Heart Fail Rev 2021;26:1103-18.

25 Bonilla-Palomas JL, Gámez-López AL, Castillo-Domínguez JC, et al. Nutritional intervention in malnourished hospitalized patients with heart failure. Arch Med Res 2016;47:535-40.

26 Yamauti AK, Ochiai ME, Bifulco PS, et al. Subjective global assessment of nutritional status in cardiac patients. Arq Bras Cardiol 2006;87:772-7.

27 Aggarwal A, Kumar A, Gregory MP, et al. Nutrition assessment in advanced heart failure patients evaluated for ventricular assist devices or cardiac transplantation. Nutr Clin Pract 2013;28:112-9. 